\title{
Écrire et relire
}

\section{Guillaume Dégé}

\section{OpenEdition}

Journals

Édition électronique

URL : https://journals.openedition.org/rbnu/3440

DOI : $10.4000 /$ rbnu.3440

ISSN : 2679-6104

\section{Éditeur}

Bibliothèque nationale et universitaire de Strasbourg

\section{Édition imprimée}

Date de publication : 1 mai 2011

Pagination : 28-39

ISSN : 2109-2761

\section{Référence électronique}

Guillaume Dégé, «Écrire et relire », La Revue de la BNU [En ligne], 3 | 2011, mis en ligne le 01 mai 2011, consulté le 08 août 2021. URL : http://journals.openedition.org/rbnu/3440 ; DOI : https://doi.org/ $10.4000 /$ rbnu. 3440

Ce document a été généré automatiquement le 8 août 2021.

\section{(c) (i) (2)(2)}

La Revue de la BNU est mise à disposition selon les termes de la Licence Creative Commons Attribution - Pas d'Utilisation Commerciale - Partage dans les Mêmes Conditions 4.0 International. 


\section{Écrire et relire}

\section{Guillaume Dégé}

\section{NOTE DE L'ÉDITEUR}

L'auteur n'ayant pas souhaité que son article soit republié numériquement en Accès Ouvert sur OpenEdition, son texte n'apparait pas ici, néanmoins vous trouverez cidessous les images illustrant ledit article, issues des collections de la Bnu. 
Albrecht Dürer, Folio 54 recto de l'Underweysung der Messung, ...

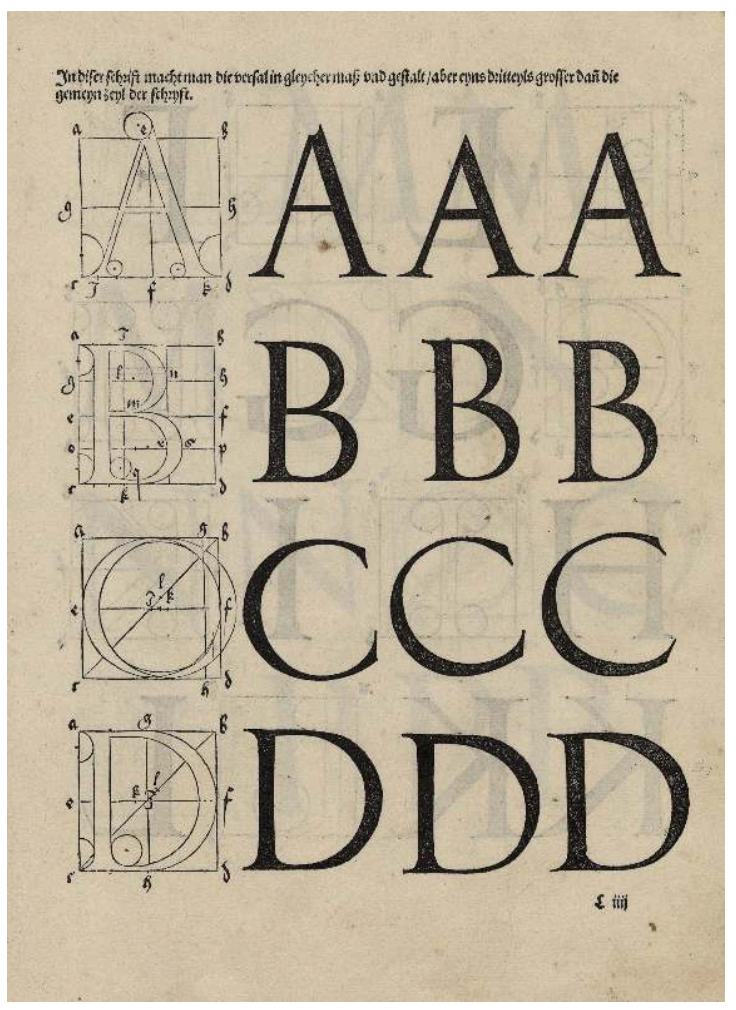

... mit dem Zirckel unn Richtscheyt..., Nuremberg, 1525

Cliché JPR-Bnu CC-BY-NC-SA

\section{Dessin anonyme (plume, lavis et aquarelle)}

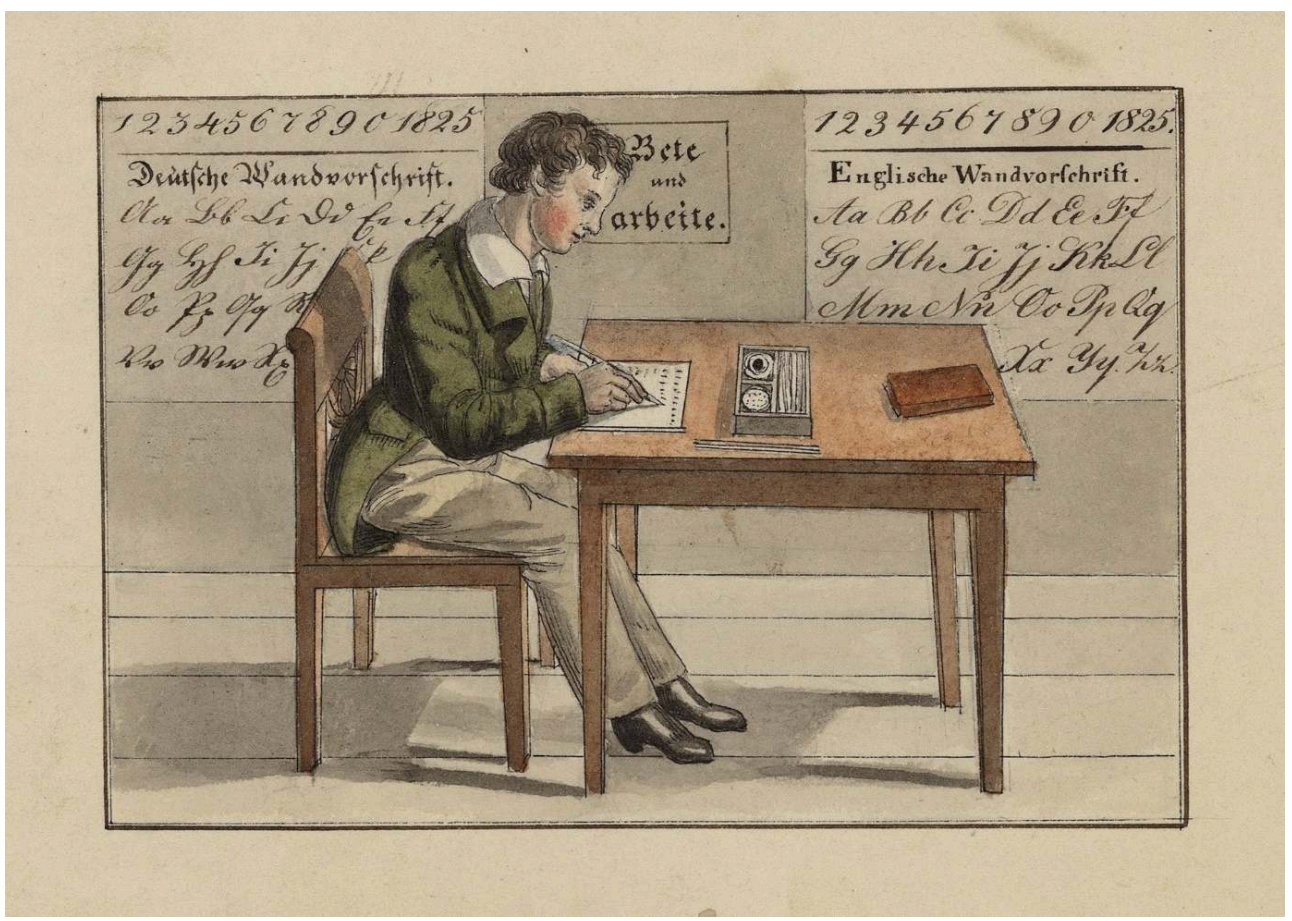

Contenu dans le recueil factice Schreibstuben - Sammlung von 9 Kupferstichen zum Thema "Schreibstube », XVIIe - XVIII siècles.

Cliché JPR-Bnu CC-BY-NC-SA 
Jean Midolle, Alphabet diabolique

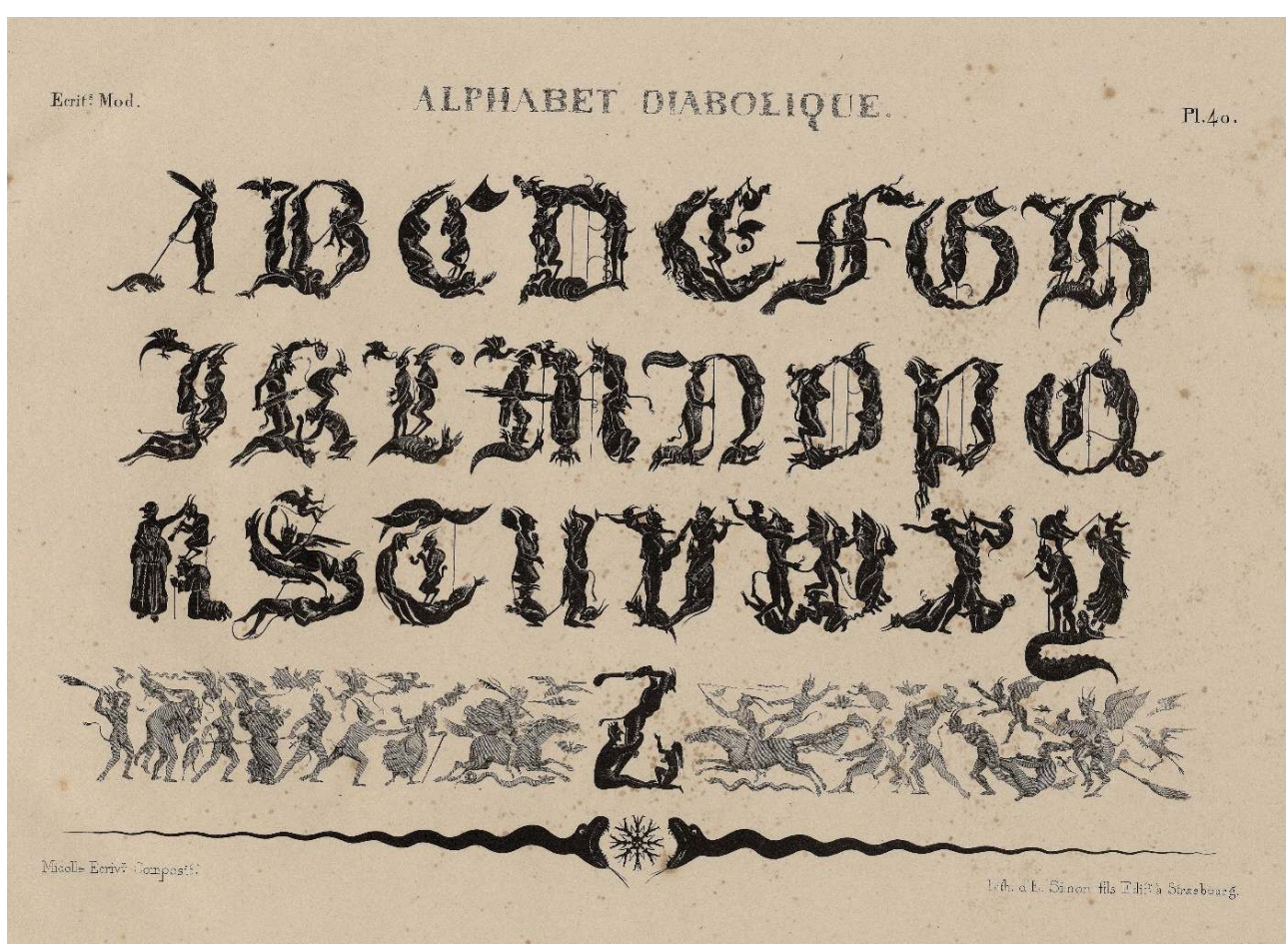

In Specimen des écritures modernes comprenant les Romaines fleuronnées, Gothiques nouvelles, Fractures, françaises, anglaises, italiennes et allemandes... - Strasbourg : Lith. E. Simon, 1834-1835

Cliché JPR-Bnu CC-BY-NC-SA 
Illustration tirée de l'ouvrage de Johann Newdörfer der Jüngere : Appendix, ...

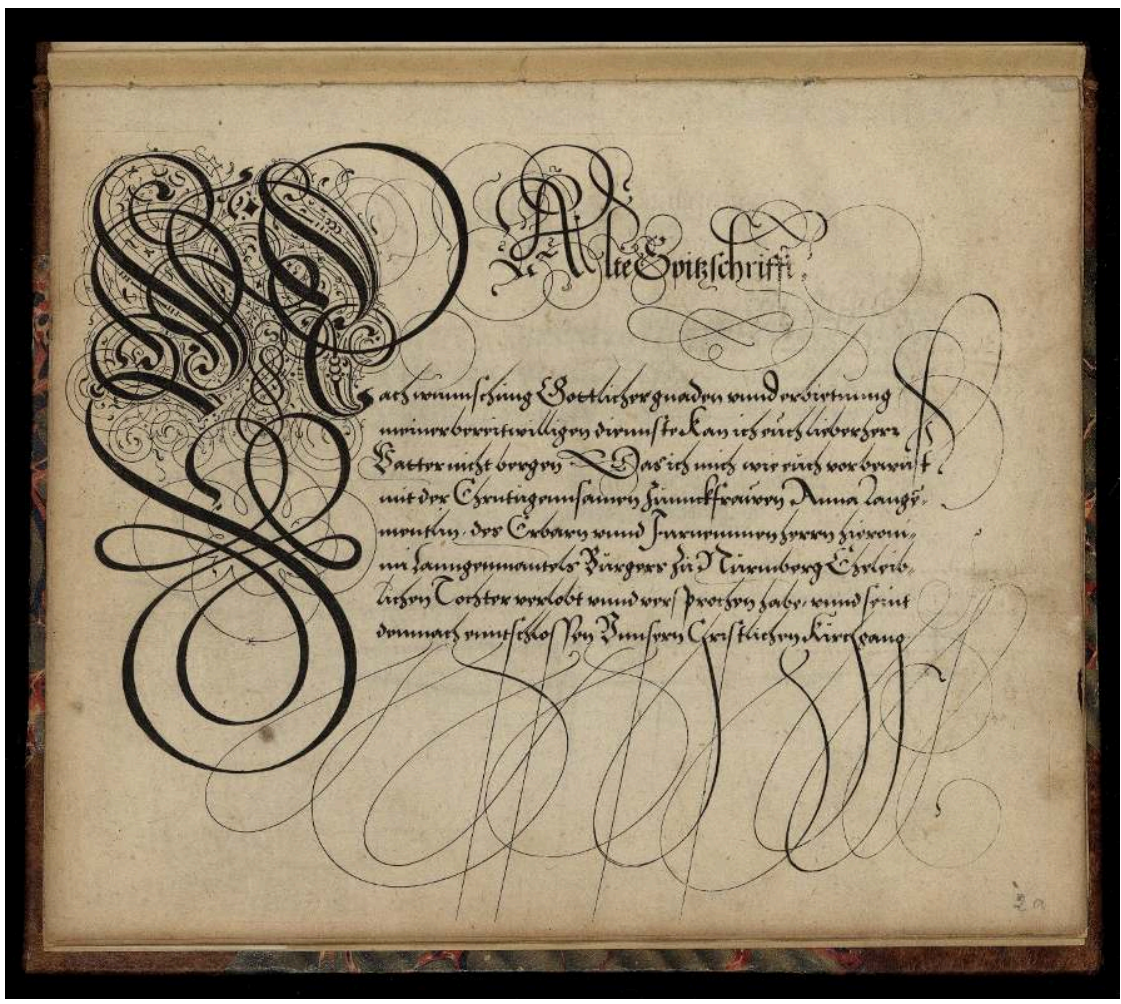

... oder kurtzer Begriff etlicher schönen Hand und anderer Schrifften zu der Newdörfferischen Schreibkunst gehörig - Nuremberg : Newdörffer, 1631 (coll. BNU)

Cliché JPR-Bnu CC-BY-NC-SA 
Jean Midolle, Galerie

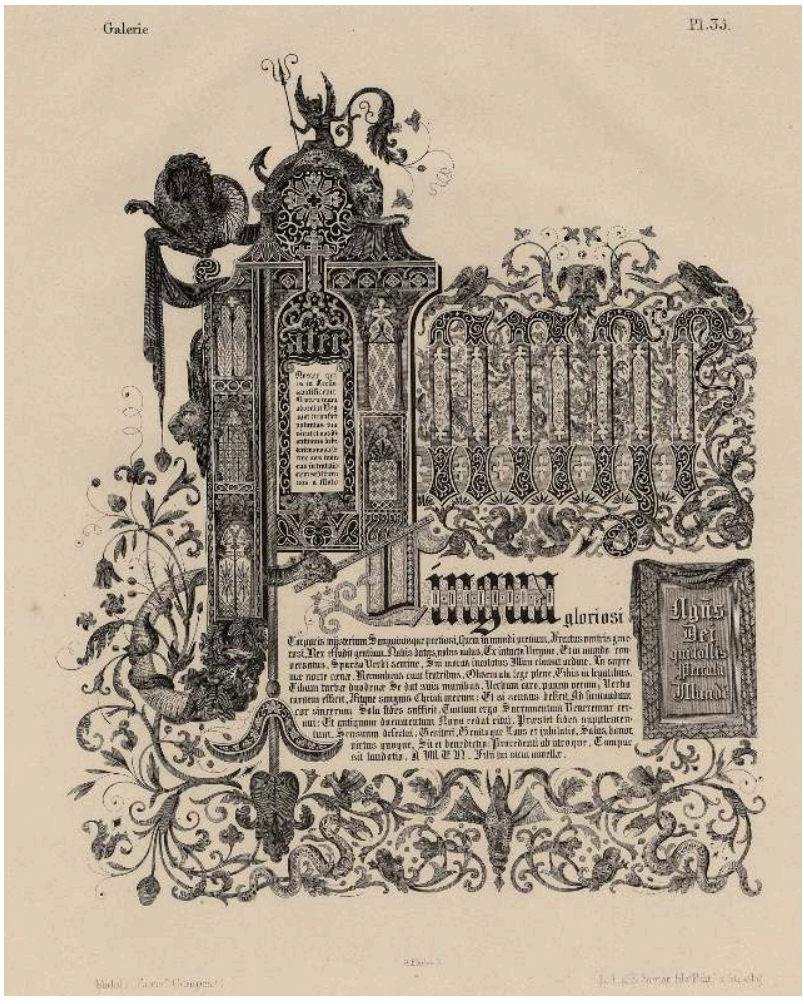

Compositions avec écritures anciennes et modernes.Strasbourg : Ed. Simon Fils, 1834-35 Cliché JPR-Bnu CC-BY-NC-SA 
Albrecht Dürer, Page 142 du De urbibus, arcibus, castelisque condentis,...

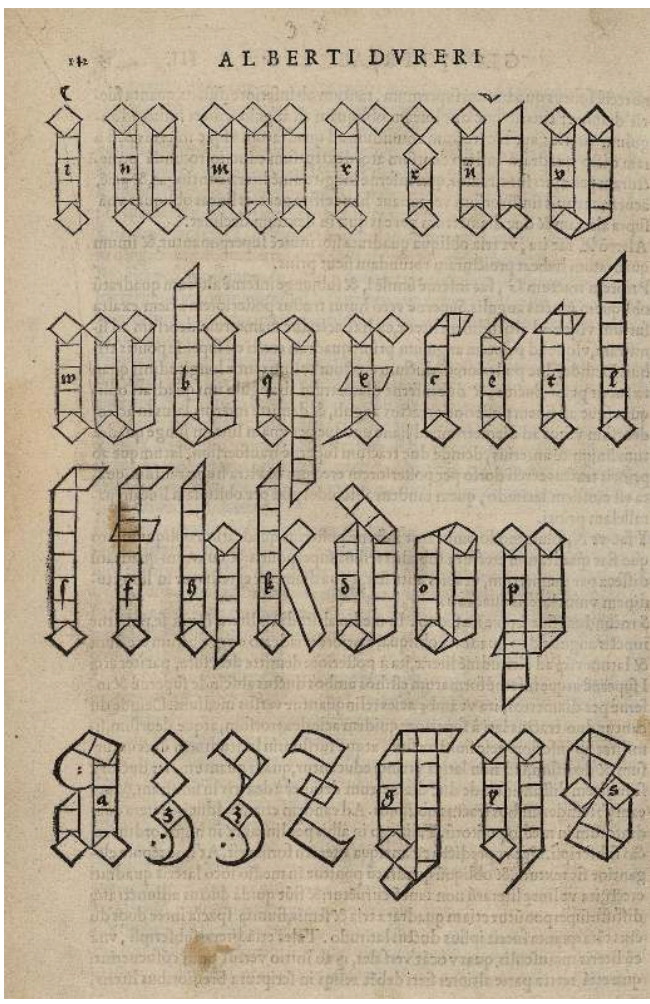

... ac muniendis rationes aliquot, praesenti bellorum necessitati accommodatissimae..., Paris, 1535 Cliché JPR-Bnu CC-BY-NC-SA

\section{AUTEUR}

\section{GUILLAUME DÉGÉ}

Professeur à l'École supérieure des arts décoratifs de Strasbourg 